\title{
High-Speed Imaging of a Laser Ablation Process Using Parallel Phase-Shifting Interferometry
}

\author{
Koichiro Yasuda ${ }^{1,2}$, Ryota Takagi ${ }^{2}$, Katsuhiro Ishii ${ }^{1}$, Kazuhisa Fujita ${ }^{1}$, Hideaki Shirai ${ }^{2}$, Akihiko Tsuboi ${ }^{1}$ \\ ${ }^{1}$ The Graduate School for Creation of New Photonics Industries, 1955-1 Kurematsu-cho, Nishi-ku, \\ Hamamatsu, Shizuoka 431-1202, Japan \\ E-mail: k.yasuda@gpi.ac.jp \\ ${ }^{2}$ Production Eng R\&D Dept, DENSO CORPORATION, 1-1, Showa-cho, Kariya, Aichi 448-8661, \\ Japan
}

\begin{abstract}
Laser-ablated plumes were characterized using a high-speed, parallel, phase-shifting interferometry to clarify influence of an expanding plume on a subsequent pulse irradiation at a high-repetition rate process. After the pulsed-laser irradiation on the surface of the work piece, the ablated material rapidly expands from the processing point. The main issue in this paper is the influence of the ejected material on the subsequent pulse incidence. To evaluate the influence of the material ejected from the work piece in an ultrashort pulse laser process, we successfully visualized the shape and characteristics of the material using a high-speed, parallel, phase-shifting interferometry. They offer a large amount of information than that in self-emission images only and could provide quantitative values, such as spatial distributions of the ejected material. Through these measurements, the effect of the ejected material on the processing result was confirmed.
\end{abstract}

DOI: $10.2961 /$ jlmn.2019.03.0005

Keywords: ultrafast laser, ablation, plume, efficiency, high-speed imaging

\section{Introduction}

Ultrashort pulse laser processing achieves high precision and high efficiency with short pulses from the range of picoseconds to femtoseconds based on recent technical development [1-3]. Thus, this technology permits many materials processing [4-6]. However, the efficiency of its process still needs improvement to be established on an industrial manufacturing scale. To establish a broad industrial application, the laser ablation process should be analyzed.

After the pulsed-laser irradiation on the surface of a work piece, the ablated material changes into plasma, vapor, and droplet and rapidly expands (i.e., plume) from the processing point [7], as shown in Fig. 1. Thereafter, the subsequent laser is irradiated at intervals of approximately several tens of microseconds, and then the plume blows out from the processing point. This phenomenon repeats during processing. At this time, if the laser is irradiated while the ejected material remains around the processing point, then the laser is absorbed and scattered by the plume. Hence, the laser energy input to the work weakens, and the processing precision and efficiency may be adversely affected [8]. Reducing the pulse interval is one of the important techniques to improve efficiency [9-10]. However, the repetition rate and efficiency are not proportional [11-14]. Thus, clarifying the relationship between the behavior of the plume and the laser is also crucial.

A number of studies have attempted to visualize the shape and characteristics of the ejected materials using various detection methods with limited success. Thus far, the shadow graph [15], Schlieren [16], and Fourier fringe analysis [17] methods have been proposed and verified. However, the shadow graph and Schlieren methods are difficult to measure quantitatively, and the Fourier fringe analysis method has a spatial resolution limit. Meanwhile, phase- shifting interferometry is used as a high-resolution interferometry, but this method cannot measure a rapid phenomenon. Until now, the plume could not be measured quantitatively [18-20].

In this study, to assess the influence of the plume during an ultrashort pulse laser process, a quantitative evaluation of the plumes using the parallel phase-shifting interferometry with a high-speed polarization camera is conducted [21]. The high-speed polarization camera can detect the highspeed fluctuation of a phase difference of the reference light and light that directly travels through the test region [22]. This study aims to establish a method that can measure the plume's refractive index and absorptivity with a spatial resolution of $5 \mu \mathrm{m}$.

In this study, to quantitatively evaluate the ultrashort pulse lase processing, we will clarify the feasibility and issues of the parallel phase-shifting interferometry as a method used for visualizing the plumes. Moreover, we analyze the results obtained by using this method, and the effect of the plume to the processing result is confirmed.

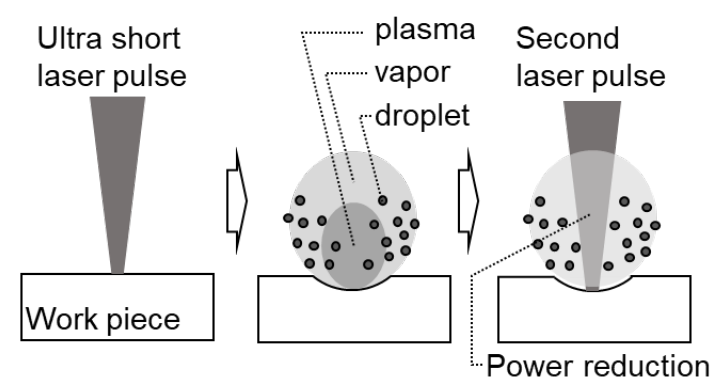

Fig. 1 Schematic of the ultrashort pulse laser processing. 


\section{Measurement system}

Phase-shifting interferometry is one of the methods used to obtain the refractive index [23]. In conventional phaseshifting interferometry, the reflection mirror is moved to change the phase of the reference light, as shown in Fig. 2 [24]. Therefore, capturing the phase-shifted images is time consuming, and measuring a rapid phenomenon is not possible.

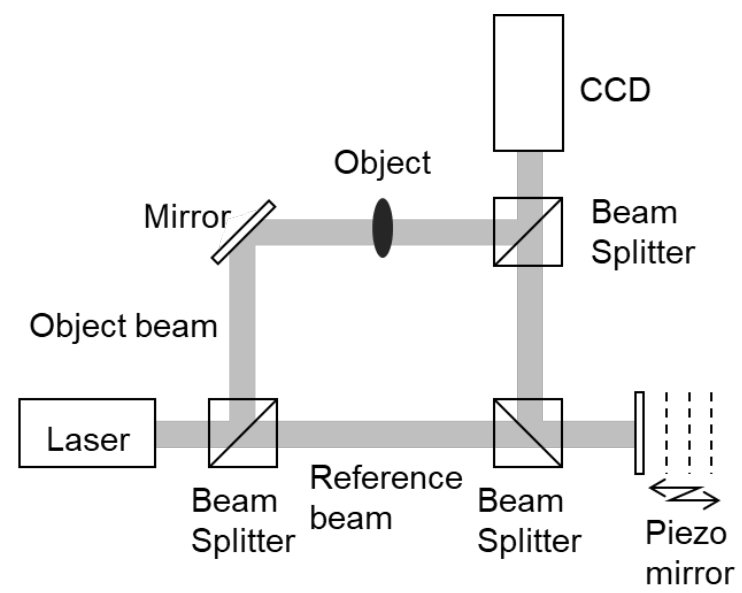

Fig. 2 Schematic of the phase-shifting interferometry.

To measure a rapid phenomenon, a single-shot acquisition of multiple phase-shifted images has been extensively investigated [25-32]. The technique that uses an array of phase-shifting elements was first reported by Horwitz et al. $[31,32]$. Millard et al. developed a polarization camera and then applied it to phase-shifting interferometry $[25,26]$. Ishikawa et al. applied the sound-field imaging generated by ultrasonic transducers driven by a pure tone of $40 \mathrm{kHz}$ [33].

Figure 3 schematically illustrates the optical configuration suitable for this study. The system is based on the polarization interferometer.

Herein, we explain the principle of the parallel phaseshifting interferometry. The Jones vectors of the reference light and the probe light are defined by

$$
E_{r}=\left[\begin{array}{c}
0 \\
E_{0 r} e^{i \Phi_{r}}
\end{array}\right],
$$

and

$$
E_{s}=\left[\begin{array}{c}
E_{0 s} e^{i \Phi_{s}} \\
0
\end{array}\right],
$$

respectively. The reference light and probe light are horizontal and vertical linearly polarized light, respectively. After passing through the quarter-wave plate whose optic axis has $45^{\circ}$ inclination, the reference and probe beams change to clockwise and counterclockwise circularly polarized light, respectively, and those Jones vectors are expressed as follows:

$$
E_{r}=\left[\begin{array}{c}
E_{0 r} e^{i\left(\Phi_{r}-\frac{\pi}{4}\right)} \\
E_{0 r} e^{i\left(\Phi_{r}+\frac{\pi}{4}\right)}
\end{array}\right]
$$

and

$$
E_{s}=\left[\begin{array}{l}
E_{0 s} e^{i\left(\Phi_{S}+\frac{\pi}{4}\right)} \\
E_{0 s} e^{i\left(\Phi_{S}-\frac{\pi}{4}\right)}
\end{array}\right] .
$$

The phase differences of the horizontal and vertical components of the two light waves are $3 \pi / 2$ and $\pi / 2$, respectively. When the base of the Jones vectors is rotated at $45^{\circ}$, they are converted as

$$
E_{r}=\left[\begin{array}{c}
E_{0 r} e^{i \Phi_{r}} \\
\left.E_{0 r} e^{i\left(\Phi_{r}+\frac{\pi}{2}\right)}\right]
\end{array}\right]
$$

and

$$
E_{s}=\left[\begin{array}{c}
E_{0 s} e^{i \Phi_{s}} \\
E_{0 s} e^{i\left(\Phi_{s}-\frac{\pi}{2}\right)}
\end{array}\right],
$$

respectively. Notably, the phase differences of the linearly polarized components at $\pm 45^{\circ}$ between the reference light and probe light are $\pi$ and 0 , respectively.

The polarization camera is necessary to capture images with different phase shifts for each polarization direction simultaneously. Figure 4 shows the schematic of the polarization camera. The camera has the phase-shifting array device in front of an image sensor, which consists of four types of phase-shifting elements whose azimuths are $0, \pi / 2, \pi$, and $3 \pi / 2$, respectively [26]. The recorded image includes four phase-shifted images, namely, $I(0), I(\pi / 2), I(\pi)$, and $I(3 \pi / 2)$, where $I(\theta)$ is the intensity of the interference fringe with phase retardation angle, $\theta$. The phase difference of the reference light and light that travels through the test region can be reconstructed using the following equation:

$$
\phi_{s}-\phi_{r}=\tan ^{-1}\left(\frac{I(3 \pi / 2)-I(\pi / 2)}{I(0)-I(\pi)}\right)
$$

Using this equation, the phase shift can be measured simultaneously.

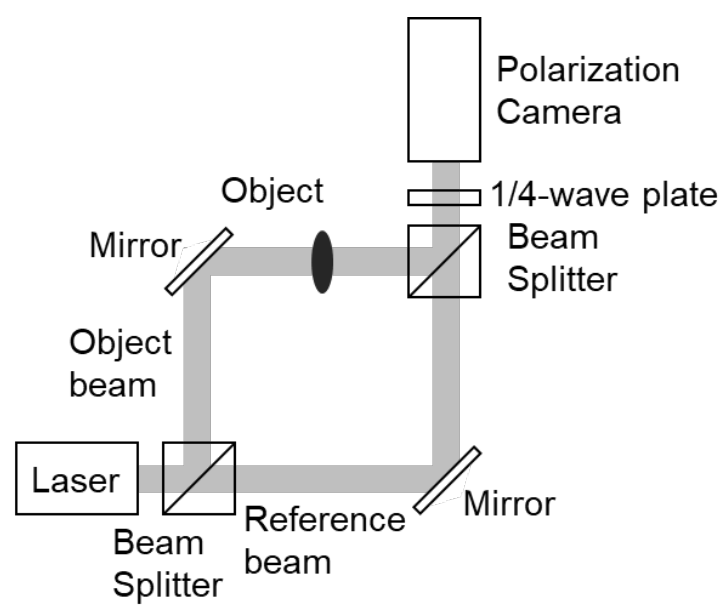

Fig. 3 Schematic of the measurement system based on the polarization interferometer.

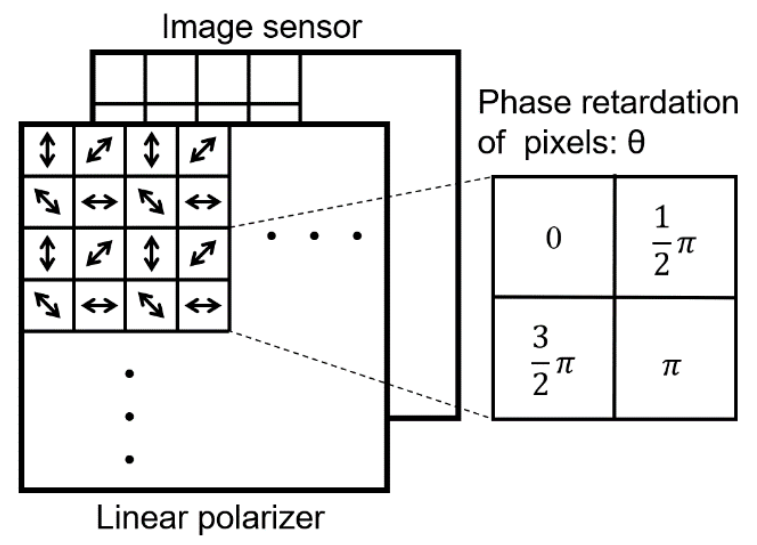

Fig. 4 Schematic of the polarization camera. 


\section{Experimental setup}

Figure 5 shows the experimental setup. The experimental setup consists of a measurement system based on phase-shifting interferometry denoted by light color and the ultrashort laser processing system represented by dark color.

In the measurement system, the linearly polarized light emitted from the oscillator enters the polarized beam splitter that divides the incident light into two orthogonally polarized light beams. One light that travels through the test region and the other one that detours because of mirrors enter the polarized beam splitter. The combined two types of light change these polarization states to clockwise and counterclockwise, respectively, by the quarter-wave plate. The two lights are captured by a high-speed polarization camera.

The laser with a wavelength of $532 \mathrm{~nm}$ is used in our instruments. A high-speed polarization camera, CRISTA made by Photron Ltd., is utilized. The maximum number of pixels of the camera is $1024 \times 1024$, which achieves the high spatial resolution of $2 \mu \mathrm{m}$ with optical lens. To capture the rapid change in the behavior of the laser processing during several tens of microseconds from the irradiation of the processing laser to the subsequent laser irradiation and determine the limitation of the frame rate, the image was set and captured with the irradiation signal of the processing laser oscillator as the trigger. The imaging timing is changed for each irradiation experiment under the same conditions, and capturing corresponding to the time change is performed. The delay time ranges from 5 to $25 \mu \mathrm{s}$, and a camera exposure time of $1 \mu$ s has been used.

In the ultrashort laser processing system, the processing laser with a wavelength of $1030 \mathrm{~nm}$ and pulse duration of 10 ps is used. The pulse energy with $180 \mu \mathrm{J}$ per 1 pulse is condensed onto a metal work at an interval of $20 \mu \mathrm{s}$. In addition, a self-luminous image is employed to compare with the conventional method.

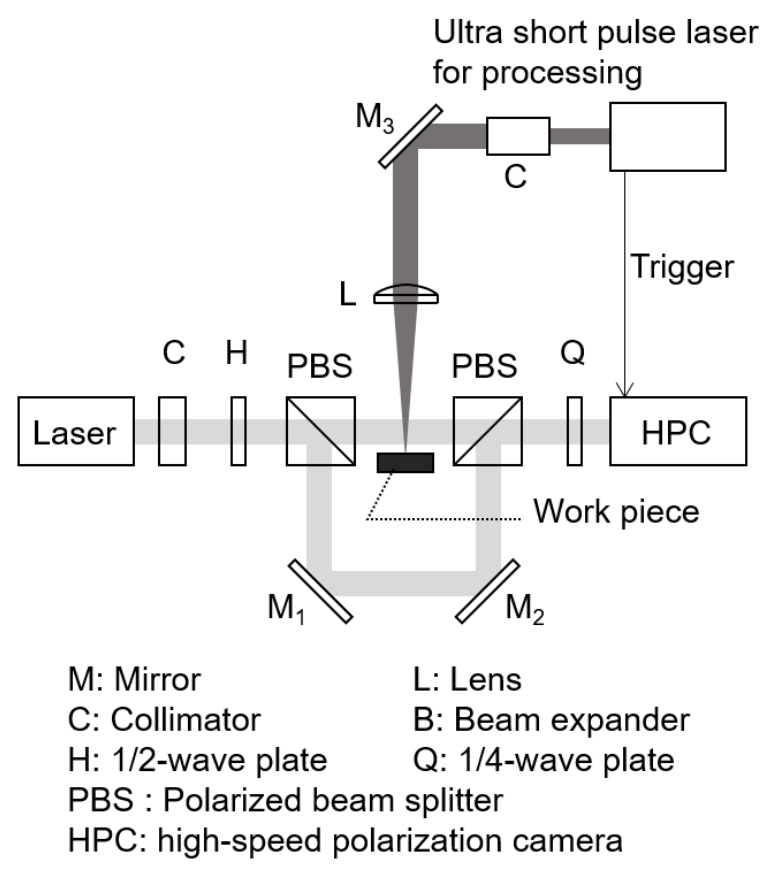

Fig. 5 Experimental setup.

The measurement system is indicated by light color, whereas the ultrashort laser processing system is denoted by dark color.

\section{Experimental results on the flat surface}

Figure 6 shows the experimental results of the basic evaluation on the flat plate. The upper part of the figure is the self-emission image, and the lower part is the phase shift image.

Immediately after the first laser irradiation, the image of the phase shift image is obtained in a wider range than the self-emission image. The red center shows the large phase change, whereas the blue edge shows the small phase change in the phase shift image. Figure 7 shows the central part of plume's distribution of the phase shift. The cross-sectional shape of the plume is evident in the figure. In the phase shift image in Fig. 6 , the plume noticeably spreads even after $5 \mu \mathrm{s}$ when self-emission is weak and difficult to observe.

Considering that the phase shift image is clearly observed, the ejection velocity of the plume can be determined quantitatively. The velocity of the plume is determined to be $77.5 \mathrm{~m} / \mathrm{s}$ by the image from immediately after the laser irradiation to $15 \mu \mathrm{s}$.

Based on the comparison between the first and second laser irradiation, when the latter is irradiated after $20 \mu \mathrm{s}$, the self-emission image shows stronger emission at a position farther from the work than the irradiation area at the former laser irradiation. As shown in the phase shift image in Fig. 6 , the irradiation of the second laser can be confirmed while the influence of the first laser's plume remains in front of the processing point. Thus, the reason of the phenomenon wherein self-emission becomes strong at the second laser irradiation is that the low-temperature plume remains in front of the work peace and the plume temperature increases in the subsequent laser.

The refractive index of the plume is calculated on the basis of the phase shift information. Figure 7 shows the distribution of the phase shift immediately after the laser irradiation.

From the phase contrast to the refractive index,

$$
n=n_{0}+\frac{h \cdot \lambda}{2 \pi \cdot d}
$$

where $n_{0}$ is the refractive index under static conditions; $h$ and $d$ are the phase contrast value and width of the phase contrast image, respectively; and $\lambda$ is the wavelength of the probe light. Using these formulas, the refractive index is determined to be $1.00044^{1}$.

In summary, capturing effects that are difficult to be observed in the self-emission image and quantitatively obtaining the refractive index are possible.

\section{Experimental results on the groove}

In the processing of products, such as grooves, the processing point becomes narrow as the processing progresses. Therefore, evaluating the behavior of the plume that ejects into a narrow space and the influence on the processing are important tasks. We attempted to visualize and quantitatively evaluate the plume during the groove shape processing, in which the shape is simulated on the basis of an actual product.

The groove shape has a width of $0.5 \mathrm{~mm}$ at the inlet side and the groove wall is $10^{\circ}$. The laser beam is irradiated 50 $\mu \mathrm{m}$ inward from the end of the groove at a focal spot diameter of $30 \mu \mathrm{m}$ and the focal position at the work piece surface 


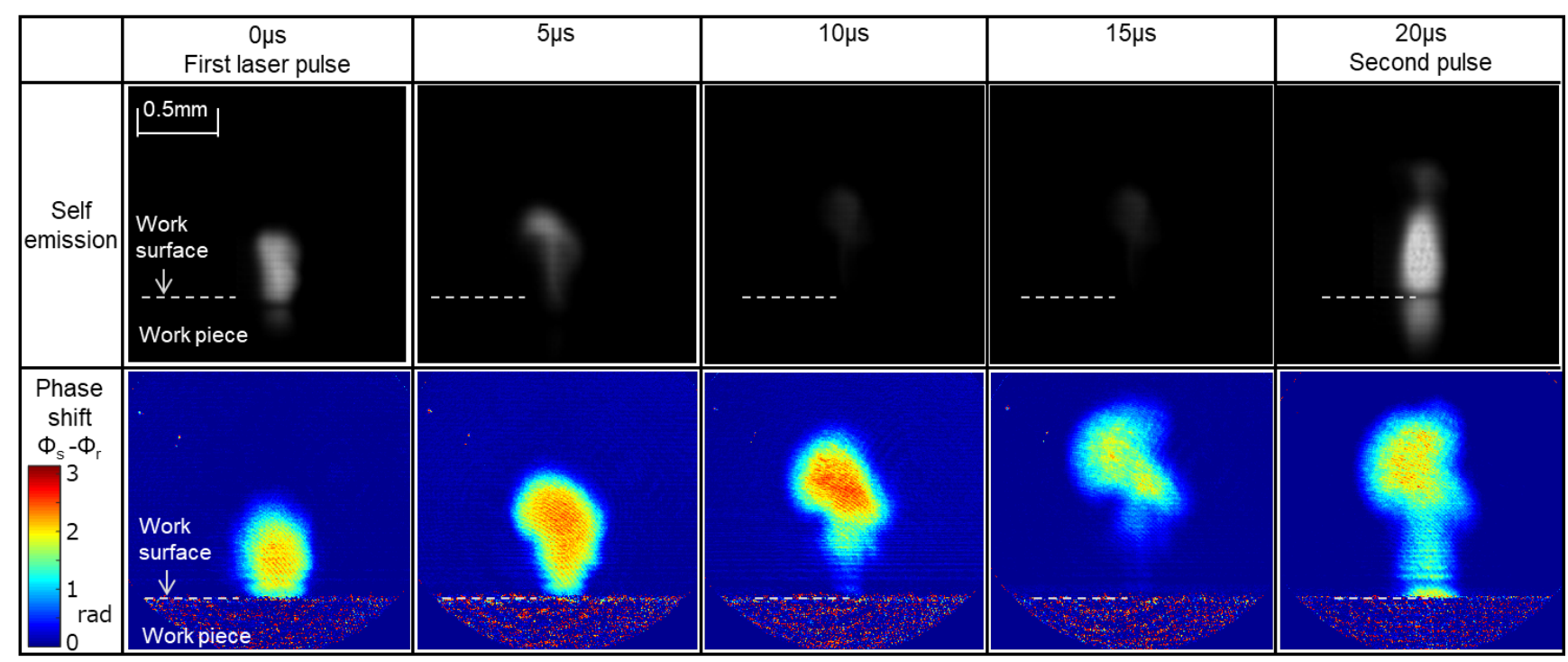

Fig. 6 Experimental result of the basic evaluation on a flat plate.
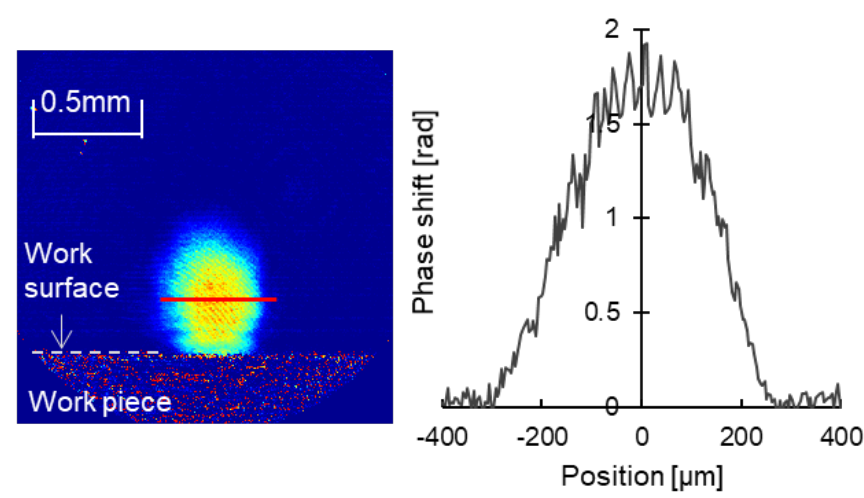

Fig. 7 Plume's distribution of the phase shift.

The condition of the laser is the same as the plate processing, and the second laser is irradiated after $20 \mu \mathrm{s}$.

The shapes of the ejected material during the first and second laser beam irradiation are compared in the flat plate and groove processing.

Figure 8 shows the experimental results. As observed in the phase contrast image at the groove processing, the ejected material is generated in the direction perpendicular to the wall. Hence, the plume is considered to generate a perpendicular force to the work surface when the material is ablated.

In addition, compared with the flat plate processing, the plume shows a wide range and the phase shift is only minimal during groove processing. The possible reason is that the energy density is reduced by the laser beam, being obliquely incident on the groove wall.

When the second laser is irradiated, the plume is confirmed to be in contact with the surface that faces the laser irradiation surface during groove processing. Moreover, the phase shift of the plume during groove processing is larger than that of flat plate processing. Figure 9 shows the evaluation results of the plume's refractive index during flat plate processing and groove processing. The $\mathrm{x}$ axis is the direction of spouting. The plume in the case of the flat plate processing advances to $1500 \mu \mathrm{m}$, whereas the plume in the groove processing advances to $580 \mu \mathrm{m}$ and is in contact with

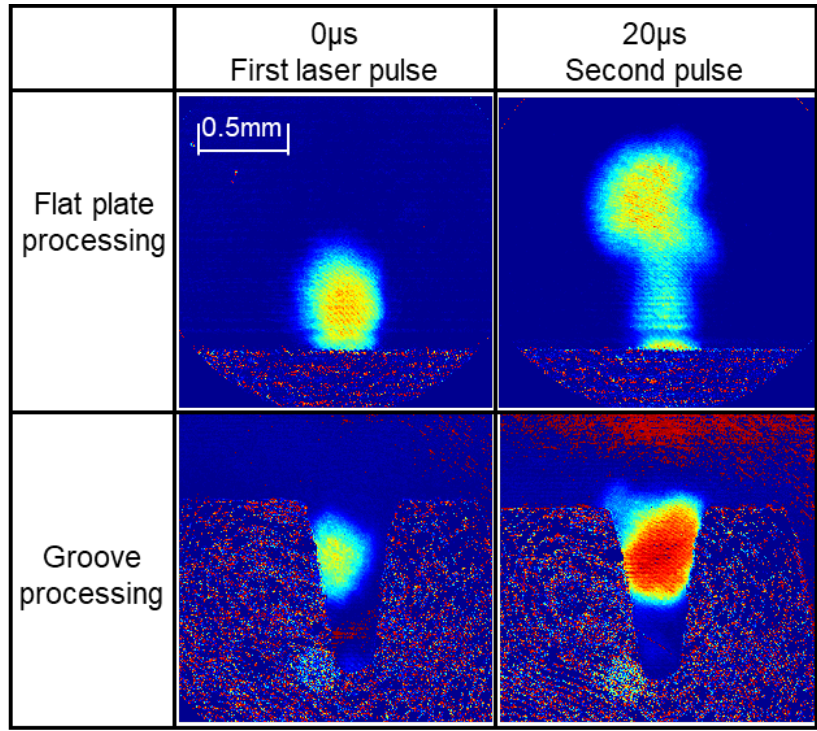

Fig. 8 Experimental results of groove processing.

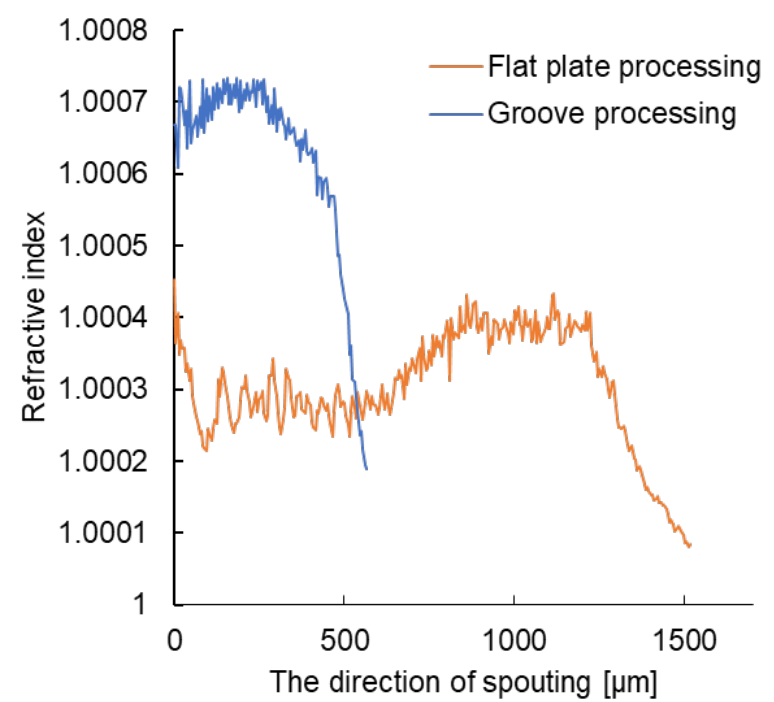

Fig. 9 Evaluation results of the ejected material's refractive index during flat plate processing and groove processing. 
the surface facing the laser irradiation surface. Thus, the increased amount of the refractive index is confirmed to be up to 1.8 times larger during groove processing than during flat plate processing.

Finally, we evaluated flat plate processing and groove processing to verify the effect of plume on processing. Figure 10 depicts the processing comparison results. In the experimental results, the average and maximum-minimum ablation rates obtained after processing is conducted 30 times are denoted in the form a range. The grooving processing results denoted a decrease in ablation rate and an increase in variation when compared with those denoted by the flat plate processing results. The laser beam refracts and scatters around the processing point because of the change in the refractive index of the processing material and the changes in energy density. In addition, the plume's shape and refractive index exhibit variations because it ejects for each pulse and interacts with each other, resulting in the large variation of the ablation rate. As shown in reference 5 of Bauerle et al., it is known that processing efficiency has various influencing factors. Based on the above results, it was clarified that the plume was one of the main factors that reduce the ablation rate.

In summary, quantitatively measuring the plume even for complex shapes close to the actual product shapes, such as groove shape, is possible. Moreover, the plume expands perpendicularly to the machining surface regardless of the irradiation direction. In the case of groove processing, the refractive index increase of the plume is up to 1.8 times the open space, and the plume stagnated in a narrow space. By comparing the ablation rates, it was observed that the ablation rate was lower and that the variation was larger in groove processing. The high refractive index suggests its remarkable influence on the processing.

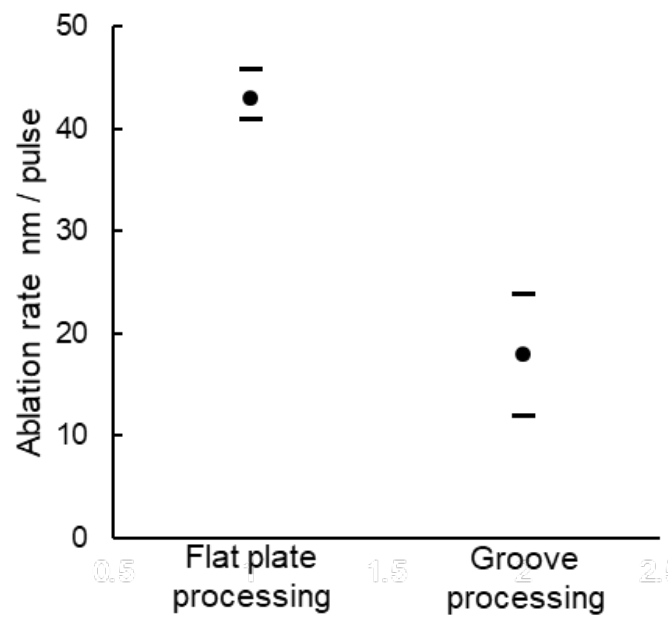

Fig. 10 Evaluation results of ablation rate comparison with and without an opposing wall surface

\section{Conclusion}

To clarify the influence of the expanding plume on the subsequent pulse irradiation in a high-repetition rate process, we developed a measurement system that can quantitatively measure the refractive index of the plume. Based on the quantitative evaluation of the behavior and the refractive index of the plume, the plume erupted in the direction perpendicular to the processing surface. Moreover, the ejection rate was $77.5 \mathrm{~m} / \mathrm{s}$ in this experiment. The plume was retained after $20 \mu$ s and considered to have an effect on the subsequent laser irradiation. When the laser was irradiated to a complex narrow space, such as groove shape, the plume had a higher refractive index than in an open space. Based on the experimental result, the refractive index increased by 1.8 times. From these measurements, the effect of the plume on the processing result was confirmed.

\section{References}

[1] B.N. Chichkov, C. Momma, S. Nolte, F. Von Alvensleben, and A. Tünnermann: Appl. Phys. A, 63, (1996) 109.

[2] L. Shah, M. Fermann, J. Dawson, and P. Barty: Opt. Express., 14, (2006) 12546.

[3] C. Momma, B.N. Chichkov, S. Nolte, F. Von Alvensleben, A. Tünnermann, H. Welling, and B. Wellegehausen: Opt. Commun., 129, (1996) 134.

[4] K. Sugioka and Y. Cheng: Appl. Phys., 1, (2014) 041303.

[5] D.W. Bäuerle: Laser processing and chemistry, (Springer, Berlin, 2013) p.279.

[6] G.L. Roth, B. Adelmann, and R. Hellmann: J. Laser Micro/Nanoengin., 10.3, (2015) 190.

[7] KH Leitz, B Redlingshöfer, Y Reg, A Otto, and M Schmidt: Physics Procedia., 12, (2011) 230.

[8] B. Neuenschwander, T. Kramer, B. Lauer, and B. Jaeggi: Proc SPIE 9350, (2015) 93500U.

[9] C Kerse, et al.: Nature, 537, (2016) 84.

[10] J. Finger, and M. Reininghaus: Opt. Express., 15, (2014) 18790.

[11]T. Kramer, B. Neuenschwander, B. Jäggi, S. Remund, U. Hunziker, and J. Zürcher: Physics Procedia., 83 (2016) 123.

[12]B. Jäggi, L. Cangueiro, D. Brunell, J. A. Ramos de Campos, C. Hairaye, and B. Neuenschwander: Proc SPIE 10519, (2018) 1051905.

[13]B. Neuenschwander, T. Kramer, B. Lauer, and B. Jaeggi: Proc SPIE 9350, (2015) 93500U.

[14] M. E. Povarnitsyn, T. E. Itina, K. V. Khishchenko, P. R. Levashov: Phys. Rev. Lett., 103, (2009) 195002.

[15] S. S. Harilal, G. V. Miloshevsky, P. K. Diwakar, N. L. LaHaye, and A. Hassanein: Phys. Plasmas., 19, (2012) 083504

[16]R Kelly, A Miotello, B Braren, and CE Otis: Appl. Phys., Lett 60, (1992) 2980.

[17]R Noll, R sattmann, V Sturm, and S Winkelmann: s. J Anal Atom Spectrom., 19, (2004) 419.

[18]B Bornschlegel and J Finger: J. Laser Micro/Nanoengin., 14.1, (2019) 88.

[19]I Mingareev and A Horn: Appl. Phys. A, 92, (2008) 917.

[20]D. J. Förster, S. Faas, S. Gröninger, F. Bauer, A. Michalowski, R. Weber, and T. Graf: Appl. Surf. Sci., (2018) 926.

[21]T Onuma and Y Otani: Opt. Commun., 315, (2014) 69.

[22] T Kakue, R Yonesaka, T Tahara, Y Awatsuji, K Nishio, S Ura, T Kubota, and O Matoba: Opt. Lett., 36.21, (2011) 4131 
[23]H Philip: 34th Annual International Technical Symposium on Optical and Optoelectronic Applied Science and Engineering, San Diego, (1990) p704.

[24] J. H. Bruning, D. R. Herriott, J. E. Gallagher, D. P. Rosenfeld, A. D. White, and D. J. brangaccio: Appl. Opt., 13. 11, (1974) 2693.

[25] M Novak, J Millerd, N Brock, M North-Morris, J Hayes, and J Wyant: Appl. Opt., 44. 32, (2005) 6861.

[26] J Millerd, N Brock, J Hayes, M North-Morris, B Kimbrough, and J Wyant: in Fringe 2005 (Springer-Verlag, 2005), p. 640.

[27]N Brock, J Hayes, B Kimbrough, J Millerd, M NorthMorris, M Novak, and J Wyant: Proc SPIE 5875, (2005) $58750 \mathrm{~F}$.

[28]R Smythe and R Moore: Opt. Eng., 23.4, (1984) 23461.

[29] O.Y. Kwon: Opt. Lett., 9.2, (1984) 59.

[30]C.L. Koliopoulos: Proc SPIE 1531, (1992) 119

[31]B.A. Horwitz and A.J. MacGovern: US patent 4575248 A (1986)

[32] J.L. McLaughlim and B.A. Horwitz: Proc SPIE 680, (1987) 35

[33] K Ishikawa, K Yatabe, N Chitanont, Y Ikeda, Y Oikawa, T Onuma, H Niwa, and M Yoshii: Opt. Express., 24.12, (2016) 12922

(Received: June 9, 2019, Accepted: November 16, 2019) 